\title{
Correlative studies on the effects of obesity, diabetes and hypertension on gene expression in omental adipose tissue of obese women
}

\author{
JN Fain
} Department of Microbiology, Immunology and Biochemistry, University of Tennessee Health Science Center,
Memphis, TN, USA

Objective: A major consequence of obesity is the enormous expansion of and enhanced inflammatory response seen in visceral adipose tissue. I hypothesized that the expression of inflammatory markers in visceral omental fat would correlate with the extent of visceral adiposity as measured by waist circumference or body mass index and that diabetes and hypertension, defined as subjects taking anti-hypertensive drugs, would be associated with changes in mRNA expression in visceral fat.

Design and methods: The expression of 106 mRNAs by RT-PCR was examined in observational studies using extracts of omental fat of obese women undergoing bariatric surgery as well as the circulating levels of some adipokines. We also compared the mRNA levels of 65 proteins in omental fat removed during gastric bypass surgery of women with and without hypertension and those with type 2 diabetes.

Results: Out of 106 mRNAs the expression of 10 mRNAs in omental fat of women not taking anti-hypertensive drugs correlated with waist circumference while 7 different mRNAs had significant correlations with circulating glucose. The correlations of waist circumference with mRNA expression were abolished, except for interleukin (IL)-1 receptor antagonist (IL-1RA), in women taking anti-hypertensive drugs. The correlations of blood glucose with omental fat mRNA expression were abolished, except for that of Akt1 and Akt2, in women taking anti-hypertensive drugs. However, the expression of 4 different mRNAs in omental fat was affected by circulating glucose in subjects taking anti-hypertensive drugs. The circulating levels of IL-1 RA, but not fatty acid binding protein 4, adipsin and phospholipase A2, correlated with both waist circumference and mRNA expression in omental fat. Conclusion: In female bariatric surgery patients, the mRNA expression of some proteins in omental fat was affected by the degree of obesity, whereas hypertension and diabetes affected a separate set of mRNAs.

Nutrition and Diabetes (2011) 1, e17; doi:10.1038/nutd.2011.14; published online 26 September 2011

Keywords: human obesity; mRNA expression; hypertension; type 2 diabetes; visceral adipose tissue; Akt1

\section{Introduction}

The current paradigm is that extreme obesity results in increased risk for hypertension and/or diabetes. ${ }^{1,2}$ The type 2 diabetes is reversible as, after weight loss of approximately $40 \mathrm{~kg}$ or more of weight due to bariatric surgery, the diabetes disappears in over $80 \%$ of humans. ${ }^{1}$ Not all extremely obese individuals develop diabetes or hypertension, and for these individuals there is no increased risk of morbidity. ${ }^{3}$ Thus, it was of interest to determine what differentiates extremely obese control women from those women with diabetes or

Correspondence: Dr JN Fain, Department of Microbiology, Immunology and Biochemistry, University of Tennessee Health Science Center, 858 Madison, Memphis, TN 38163, USA.

E-mail: jfain@uthsc.edu

Received 18 July 2011; accepted 11 August 2011 hypertension with regard to gene expression in omental fat. One study found that half of the extremely obese women with a mean waist circumference of $102 \mathrm{~cm}$ developed diabetes mellitus/hypertension, and their visceral fat mass was $26 \%$ greater than those not at risk with a waist circumference that averaged $96 \mathrm{~cm} .{ }^{4}$ Since these women had more visceral fat, this could account for the differences in diabetes/hypertension. There is increasing evidence that the accumulation of visceral omental fat is associated with the development of diabetes/hypertension. ${ }^{5}$ It is recognized that waist circumference is an effective and inexpensive measure of visceral fat accumulation. ${ }^{6-10}$ Waist circumference in women is a better predictor of coronary heart disease than is body mass index (BMI) ${ }^{8-10}$ and correlates with visceral fat accumulation as measured by magnetic resonance imaging, ${ }^{6}$ dual-emission $\mathrm{x}$-ray absorptiometry ${ }^{7}$ or fat mass measured by bioelectrical impedance. ${ }^{11}$ Thus, it is 
important to examine extremely obese women without diabetes or hypertension but with similar waist circumferences to those with diabetes or hypertension.

The present studies focus on the visceral omental fat of women because (1) it has a key role in the pathogenesis of the deleterious metabolic consequences of obesity and (2) women comprise 80 to $90 \%$ of bariatric surgery patients and (3) most intra-abdominal fat is omental fat. The omentum also has a central role in an inflammatory response that involve macrophages in defending against peritonitis. ${ }^{12}$ In obesity per se, this macrophage infiltration into the omentum may result in an enhanced inflammatory response that promotes insulin resistance and ultimately to diabetes/ hypertension, and it has been reported that omentectomy in connection with open bariatric surgery resulted in an enhanced insulin sensitivity as compared with patients undergoing open bariatric surgery without omentectomy. ${ }^{13}$

The effect of waist circumference on the mRNA expression of 106 proteins with known functions or that have been linked to obesity was examined in visceral omental adipose tissue of obese women in the present observational study. The correlations of waist circumference and BMI with the circulating levels of 18 of these proteins were also examined in obese females as well as the effects of DM and hypertension on the mRNA expression of 61 proteins. Hypertension was examined by comparing subjects on anti-hypertensive drugs and DM defined as those with fasting blood glucose values over $125 \mathrm{mg} \mathrm{dl}^{-1}$.

Quantification of the mRNA was based on qRT-PCR analysis rather than gene microarrays because of the large number of subjects and the desire to examine only known proteins with established roles in adipose tissue metabolism. The proteins whose expression was examined either were pro-inflammatory adipokines (apelin, amyloid A, interleukin-1 $\beta$ (IL-1 $\beta$ ), IL-6, lipocalin-2, macrophage migration inhibitory factor, monocyte chemoattractant protein 1 , osteoprotegerin, soluble phospholipase $\mathrm{A}_{2}\left(\mathrm{sPLA}_{2}\right)$, regulated on activation, normal $\mathrm{T}$ cell expressed and secreted (RANTES), tumor necrosis factor- $\alpha$ ), anti-inflammatory adipokines (scavenger receptor for hemoglobin/haptoglobin complexes (CD163), IL-1 receptor antagonist, IL-10, nerve growth factor), serpins (serpin 1 also known as plasminogen activator inhibitor 1) or visceral adipose tissue-derived serine protease inhibitor)), those implicated in the regulation of inflammation (CD14, CD150/signaling lymphocytic activation molecule family member 1 , hypoxia-inducible factor $1 \alpha$, $11 \beta$ hydroxysteroid dehydrogenase-1 (11ßHSD1), NF- $\kappa$ B p50 subunit, Toll-like receptor 4 (TLR4), tumor progression locus $2 /$ mitogen-activated protein kinase 8 /cotyledon trichome 1 ), oxidative stress and/or reactive oxygen species (cytochrome $C$ oxidase subunit Vib polypeptide 1 ), glutathione peroxidase 3, glutathione $S$-transferase A4, p67 component of $\mathrm{NADPH}$ oxidase, endothelial nitric oxide synthase, mitochondrial superoxide dismutase-2 tv1, sirtuin-1), angiogenesis/ endothelial cell function (endothelin-1, vascular endothelial growth factor A (VEGF-A), VEGF receptor (VEGFR1 or
VEGFR2), hypertension (angiotensin I converting enzyme, angiotensin II receptor-1, angiotensin II receptor-2, angiotensinogen, renin receptor), regulation of metabolism and/ or gene transcription (AMP-activated protein kinase $\alpha 2$ catalytic subunit, peroxisome proliferator activator receptor- $\gamma$ coactivator $1 \alpha$, phosphatase and tensin homolog, phosphoinositide (PI)-3 kinase catalytic $\alpha$ subunit (PI-3 kinase), PR domain-containing 16, sirtuin-1, suppresssor of cytokine signaling molecule 1 , tribbles $3,1 \alpha, 25$-dihydroxyvitamin $\mathrm{D}$ hydroxylase ( $1 \alpha$-hydroxylase)) or those preferentially expressed with known functions in adipocytes (adiponectin, adipsin, Akt-1 (protein kinase B1), Akt2 (protein kinase B2, cell death-inducing DFFA (DNA fragmentation factor-alpha)-like effector A (CIDEA), fatty acid translocase/CD36, fatty acid binding protein 4 (FABP4), insulin receptor tv1, lipoprotein lipase, perilipin, peroxisome proliferator-activated receptor-gamma, retinol binding protein 4, stearoyl CoA desaturase1, uncoupling protein 2, zinc $\alpha 2$ glycoprotein).

\section{Materials and methods}

Abdominal visceral omental adipose tissue was removed from extremely obese women undergoing laparoscopicadjustable gastric banding (lap band) surgery or gastric bypass with Roux-en-Y gastroenterostomy surgery as well as women who were undergoing abdominoplasty surgery approximately a year after gastric bypass surgery. This was an observational study involving non-smoking women undergoing bariatric surgery in a private practice setting in Memphis, Tennessee, USA. Approximately 44\% self-identified themselves as African American, and we have previously reported little differences in omental fat metabolism between the Caucasian and African-American women. ${ }^{11}$ As men comprised only $16 \%$ of the bariatric surgery patients, their results were not included in this study because of insufficient numbers for comparison.

The studies shown in Tables 1 and 3 used omental fat derived from women undergoing gastric bypass or lap band surgery or abdominoplasty. However, the omental fat samples used for the studies shown in Tables 2 and 4 were obtained from a subset of the women who were undergoing only gastric bypass or lap band surgery. In the present studies, hypertensive women undergoing gastric bypass or lap band surgery are defined as those who listed on their hospital admission forms that they were taking antihypertensive agents. Approximately $63 \%$ of the women were taking angiotensin-converting enzyme or angiotensin receptor antagonists, 54\% diuretics, $14 \% \mathrm{Ca}^{++}$channel antagonists and 26\% $\beta$-adrenergic antagonists. About $20 \%$ were taking two or more drugs and $9 \%$ three or more drug types, whereas only $22 \%$ of the women were taking only diuretics. The diabetic women were those who indicated that they were taking insulin or drugs for the treatment of 
Table 1 Correlation coefficients in omental fat comparing waist circumference and BMI with mRNA expression in control women and those taking anti-hypertensive drugs

\begin{tabular}{|c|c|c|c|c|c|c|}
\hline \multirow[t]{2}{*}{$\begin{array}{l}m R N A, B M I \text { or } \\
\text { blood glucose }\end{array}$} & \multicolumn{3}{|c|}{ Controls } & \multicolumn{3}{|c|}{$\begin{array}{c}\text { Women taking } \\
\text { anti-hypertensive drugs }\end{array}$} \\
\hline & $n$ & r-value & P-value & $\mathrm{n}$ & r-value & P-value \\
\hline & \multicolumn{6}{|c|}{ Waist circumference } \\
\hline Amyloid A & 37 & 0.57 & 0.001 & 31 & 0.31 & 0.09 \\
\hline p67phox & 36 & 0.53 & 0.001 & 38 & 0.05 & 0.74 \\
\hline PAl-1 & 25 & 0.58 & 0.002 & 20 & 0.15 & 0.54 \\
\hline 11ßHSD1 & 44 & 0.47 & 0.001 & 45 & 0.17 & 0.26 \\
\hline IL-1RA & 41 & 0.45 & 0.003 & 30 & 0.42 & 0.021 \\
\hline Leptin & 47 & 0.48 & 0.001 & 37 & 0.21 & 0.20 \\
\hline Perilipin & 40 & 0.33 & 0.050 & 43 & 0.12 & 0.42 \\
\hline Pl-3 kinase & 38 & -0.36 & 0.029 & 26 & -0.08 & 0.71 \\
\hline CIDEA & 49 & -0.27 & 0.059 & 49 & -0.29 & 0.046 \\
\hline TLR4 & 42 & -0.37 & 0.016 & 41 & 0.00 & 0.98 \\
\hline BMI & 71 & 0.87 & 0.0001 & 63 & 0.81 & 0.0001 \\
\hline \multirow[t]{2}{*}{ Glucose } & 53 & 0.22 & 0.11 & 54 & 0.27 & 0.044 \\
\hline & \multicolumn{6}{|c|}{ Basal metabolic index (BMI) } \\
\hline Amyloid A & 37 & 0.57 & 0.001 & 33 & 0.22 & 0.21 \\
\hline p67phox & 37 & 0.39 & 0.017 & 39 & -0.09 & 0.59 \\
\hline PAl-1 & 25 & 0.48 & 0.015 & 20 & 0.14 & 0.55 \\
\hline 11ßHSD1 & 45 & 0.32 & 0.029 & 45 & 0.19 & 0.22 \\
\hline IL-1RA & 42 & 0.27 & 0.09 & 30 & 0.19 & 0.32 \\
\hline Leptin & 48 & 0.22 & 0.12 & 37 & 0.16 & 0.33 \\
\hline Perilipin & 41 & 0.16 & 0.32 & 43 & 0.12 & 0.46 \\
\hline Pl-3 kinase & 39 & -0.23 & 0.15 & 26 & -0.09 & 0.67 \\
\hline CIDEA & 50 & -0.35 & 0.012 & 49 & -0.31 & 0.033 \\
\hline TLR4 & 43 & -0.35 & 0.022 & 41 & -0.12 & 0.47 \\
\hline Glucose & 53 & 0.22 & 0.12 & 54 & 0.31 & 0.020 \\
\hline
\end{tabular}

Abbreviations: CIDEA, cell death-inducing DFFA (DNA fragmentation factoralpha)-like effector A; IL-1RA, interleukin (IL)-1 receptor antagonist; PAI-1, plasminogen activator inhibitor type 1; PI-3, phosphoinositide-3; TLR-4, Tolllike receptor; $11 \beta$ HSD1, $11 \beta$ hydroxysteroid dehydrogenase- 1 . The Pearson correlation coefficients for the indicated mRNAs were obtained using BMI or waist circumference for the number of different female bariatric patients divided into controls, those not taking anti-hypertensive drugs and those taking hypertensive drugs. The number of subjects in each group is under $n$. Omental fat was derived from women undergoing gastric bypass surgery, lap band surgery or abdominoplasy. The mRNAs whose expression is at least threefold greater in fat than in non-fat/stromal cells of omental adipose tissue $^{16}$ are shown in bold.

diabetes and who had fasting blood glucose values above $125 \mathrm{mg} \mathrm{dl}^{-1}$ before surgery. In the studies shown in Tables 1 , 3 and 4 , the control group included diabetic women taking hypertensive drugs, whereas the diabetic women taking antihypertensive drugs were included with the non-diabetic women taking anti-hypertensive drugs. Bariatric surgery was limited to patients with their diabetes under reasonable control, and no subject from whom omental fat was obtained had a fasting blood glucose value above $250 \mathrm{mg} \mathrm{dl}^{-1}$. Of the subjects whose fat was obtained for the studies shown in Table 2 the women with diabetes were those unable to get their fasting blood glucose values below $125 \mathrm{mg} \mathrm{dl}^{-1}$ during the period before surgery and $\sim 70 \%$ were women taking anti-hypertensive drugs. About half of the diabetic subjects who indicated that they were taking drugs and or insulin upon hospital admission were able to get their blood glucose values below $125 \mathrm{mg} \mathrm{dl}^{-1}$ before surgery. These women were included with the controls after data analysis indicated that the effects of DM on gene expression were only seen in those subjects unable to get their fasting blood glucose below $125 \mathrm{mg} \mathrm{dl}^{-1}$. Each experimental replication involved tissue from a separate individual. The study had the approval of the University of Tennessee Health Science Center Institutional Review Board, and all patients involved gave their informed consent.

The isolation of RNA and assay of mRNA involved realtime qPCR as previously described. ${ }^{14}$ The cDNA was prepared using the Transcriptor First Strand cDNA synthesis Kits from Roche Diagnostics (Indianapolis, IN, USA) using $1 \mu \mathrm{g}$ of total RNA determined by absorption at $260 \mathrm{~nm}$. The quantification of cDNA was accomplished using the Roche Lightcycler 480 Real-time RT-PCR system and their Universal Probe Library of short hydrolysis Locked Nucleic Acid dual hybridization probes in combination with the primers suggested by their web-based assay design center (http:// www.universalprobelibrary.com). Integrated DNA Technologies (Coralville, IA, USA) synthesized the primers. In each assay, cDNA derived from 70 ng of total RNA was used and the ratio of the right to left primers was 1 . Relative quantification of the data was based on the ratio of each mRNA to that of cyclophilin A that served as the endogenous control (reference gene) in each run. The mean cyclophilin A $C p$ value was 25.8 (s.d. was 1.2) for 90 women and ranged from 23.3 to 29.5 .

Statistical analyses were carried out with Student's $t$-test. The Pearson correlation coefficients were determined using the GraphPad Prism program (San Diego, CA, USA), assuming a Gaussian population and a two-tailed $P$-value.

\section{Results}

Correlations between gene expression in omental fat and waist circumference or BMI

The qRT-PCR procedure for mRNA quantification generally involves use of the same amount of total RNA in each run and normalization to a so-called reference or housekeeping gene. ${ }^{15}$ We used the same amount of total RNA in each run and cyclophilin A as our reference gene. It is important to demonstrate that obesity does not affect its mRNA expression. We therefore examined cyclophilin mRNA expression in 95 subjects, each value was the mean of 4 to 10 runs, and found no correlation with waist circumference (the Pearson correlation coefficient $r$ was -0.002).

The question arises as to whether women taking antihypertensive drugs differed from control or diabetic women with respect to mRNA expression in omental fat. I compared the Pearson correlation coefficients for waist circumference or BMI versus mRNA expression of 106 proteins in visceral omental fat using omental fat samples from 27 to 51 control women and 18 to 47 women taking anti-hypertensive drugs. 
Table 2 Comparison of age, waist, fat mass and 65 mRNAs in omental fat from extremely obese control women versus those with diabetes or those taking drugs for treatment of hypertension

\begin{tabular}{|c|c|c|c|}
\hline & $\begin{array}{l}\text { Controls } \\
(\mathrm{n}=56)\end{array}$ & $\begin{array}{l}\text { Hypertensives } \\
\quad(\mathrm{n}=32)\end{array}$ & $\begin{array}{c}\text { Diabetics } \\
(\mathrm{n}=17)\end{array}$ \\
\hline Age (in years) & $39.4 \pm 1.3$ & $40.6 \pm 1.6$ & $46.1 \pm 2.9^{*}$ \\
\hline Waist circumference (in $\mathrm{cm}$ ) & $128 \pm 1.9$ & $125 \pm 2.5$ & $133 \pm 5.0$ \\
\hline BMI & $49.1 \pm 1.0$ & $48.5 \pm 3.2$ & $54.2 \pm 2.3^{*}$ \\
\hline Fat mass (in kg) & $69.5 \pm 2.4$ & $68.7 \pm 3.2$ & $82.6 \pm 6.4$ \\
\hline Glucose (in $\mathrm{mg} \mathrm{dl}^{-1}$ ) & $95.3 \pm 1.9$ & $88.8 \pm 1.5^{* *}$ & $159 \pm 7.8^{\star \star \star}$ \\
\hline
\end{tabular}

mRNAs altered in subjects with diabetes or taking drugs for treatment of hypertension

Akt1 (protein kinase B1)

Akt2 (protein kinase B2)

Apelin

p67phox component of NOX2

Renin receptor

CD150/SLAMF-1

Toll-like receptor 4 (TLR4)

VEGFR1

\begin{tabular}{|c|c|c|}
\hline $0.62 \pm 0.20$ & $0.61 \pm 0.26$ & $-1.39 \pm 0.30^{* *}$ \\
\hline $2.11 \pm 0.1$ & $1.82 \pm 0.16$ & $1.10 \pm 0.22^{* * *}$ \\
\hline $535+01$ & $-5.91 \pm 0.18^{*}$ & $-5.15 \pm 0.22$ \\
\hline$-1.38 \pm 0.18$ & $-1.56 \pm 0.19$ & $-1.87 \pm 0.12^{*}$ \\
\hline $.52 \pm 0.13$ & $1.12 \pm 0.21^{\star *}$ & 0.6 \\
\hline$-4.15 \pm 0.24$ & $-3.15 \pm 0.29^{\star *}$ & $-4.54 \pm 0.41$ \\
\hline$-7.00 \pm 0.42$ & $-7.63 \pm 0.35$ & $-8.62 \pm 0.40^{* *}$ \\
\hline $036+$ & $-0.83 \pm 0.15^{*}$ & $-1.35 \pm 0.34^{\star *}$ \\
\hline & $1.01 \pm 0.15$ & $0.73 \pm 0.10^{*}$ \\
\hline
\end{tabular}

mRNAs unaltered in subjects with diabetes or taking drugs for treatment of hypertension

ACE

Adiponectin

Adipsin

AMPK $\alpha 2$ catalytic subunit

Amyloid A

Angiotensin II receptor-2

Angiotensin II receptor-1

Angiotensinogen

CD-14

CD-163

CIDEA

Cytochrome $C$ oxidase

Endothelin-1

FABP-4

FAT/CD36

GPX3

Glutathione S-transferase A4

25-hydroxyvitamin D $1 \alpha$

hydroxylase

Hypoxia-inducible factor

$1 \alpha(\mathrm{HIF} 1 \alpha)$

11- $\beta$ HSD 1

Interleukin $1 \beta$ (IL-1 $\beta)$

Interleukin 1 receptor

antagonist (IL-1RA)

IL-6

IL-10

Insulin receptor

Leptin

Lipocalin-2

Lipoprotein lipase

Monocyte chemoattractant

protein 1 (MCP-1)

MIF

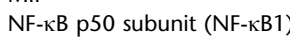

Nerve growth factor (NGF)

eNOS

Osteoprotegerin

PAI-1

Perilipin

PGC- $1 \alpha$

Phospholipase A2

PPAR $\gamma$

PR domain containing 16 tv 1

RANTES

PI 3 kinase catalytic $\alpha$ subunit

\begin{tabular}{|c|c|c|}
\hline & & \\
\hline $3.99 \pm 0.21$ & $3.81 \pm 0.27$ & $4.01 \pm 0.28$ \\
\hline $2.45 \pm 0$. & $2.78 \pm 0.19$ & $1.94 \pm 0.28$ \\
\hline$-2.91 \pm 0.15$ & $-2.73 \pm 0.21$ & $-2.96 \pm 0.16$ \\
\hline $5.85 \pm 0$. & $5.40 \pm 0.42$ & $5.36 \pm 0.40$ \\
\hline$-8.48 \pm 0.35$ & $-8.26 \pm 0.37$ & $-8.14 \pm 0.49$ \\
\hline $0.17 \pm 0.12$ & $0.70 \pm 0.28$ & $0.32 \pm 0.29$ \\
\hline$-3.29 \pm 0$. & $-3.46 \pm 0.24$ & $-3.60 \pm 0.37$ \\
\hline & $0.52 \pm 0.23$ & $0.60 \pm 0.45$ \\
\hline$-0.35 \pm 0.35$ & $-0.74 \pm 0.26$ & $-0.33 \pm 0.35$ \\
\hline $0.48 \pm 0$. & $0.48 \pm 0.23$ & $0.39 \pm 0.2$ \\
\hline $0.65 \pm 0$. & $0.38 \pm 0$ & $0.58 \pm 0.10$ \\
\hline$-2.41 \pm 0$. & $-2.27 \pm 0.20$ & $-2.84 \pm 0.24$ \\
\hline $7.84 \pm 0$. & $7.91 \pm 0.20$ & $7.64 \pm 0.12$ \\
\hline $6.52 \pm 0$ & 6.18 & $6.45 \pm 0.45$ \\
\hline 4.18 & 4.2 & $3.39 \pm 0.65$ \\
\hline-1.06 & -1.5 & -0.8 \\
\hline-1.14 & -0.1 & -1.3 \\
\hline $0.01 \pm 0$ & $0.40 \pm 0.28$ & \\
\hline 0.5 & 0. & -0.0 \\
\hline-3.1 & -2.1 & -2.9 \\
\hline 4.5 & \pm 0.30 & $-4.32 \pm 0$ \\
\hline-9.21 & -8.7 & -8.6 \\
\hline-2.48 & \pm 0.27 & -2.6 \\
\hline$-1.22 \pm 0$ & \pm 0.44 & $-1.56 \pm 0.40$ \\
\hline $2.32 \pm 0$ & \pm 0 & $1.63 \pm 0.30$ \\
\hline$-8.97 \pm 0$ & $9 \pm 0.73$ & $-8.80 \pm 0.56$ \\
\hline $4.02 \pm 0$ & $4.16 \pm 0.28$ & $3.62 \pm 0.3$ \\
\hline $2.54 \pm 0$. & $2.69 \pm 0.22$ & $2.26 \pm 0.6$ \\
\hline-2.7 & -2.2 & -2 \\
\hline$-3.06 \pm 0$. & $-2.61 \pm 0$ & $-3.06 \pm 0$ \\
\hline$-1.93 \pm 0$. & $-1.94 \pm 0$ & $-1.89 \pm 0$ \\
\hline-2.4 & -2.3 & $-2.16 \pm 0.2$ \\
\hline-3.6 & $-4.11 \pm 0$ & $-3.63 \pm 0.2$ \\
\hline$-1.85 \pm 0$ & $-1.03 \pm 0.67$ & $-1.78 \pm 0.7$ \\
\hline & 2. & $2.28 \pm 0.3$ \\
\hline$-3.46 \pm 0$ & $-3.21 \pm 0.19$ & $-3.25 \pm 0.2$ \\
\hline $1.33 \pm 0.35$ & $1.65 \pm 0.35$ & $1.61 \pm 0.3$ \\
\hline $1.61 \pm 0$. & $1.50 \pm 0$ & $1.58 \pm 0.2$ \\
\hline$-4.84 \pm 0.18$ & $-4.68 \pm 0.25$ & $-4.91 \pm 0.2$ \\
\hline & $0.48 \pm 0.20$ & $0.18 \pm 0.3$ \\
\hline $0.15 \pm 0.14$ & $0.01 \pm 0.25$ & $-0.42 \pm 0.2$ \\
\hline
\end{tabular}

Table 2 (continued)

\begin{tabular}{lrrr}
\hline & $\begin{array}{c}\text { Controls } \\
(\mathrm{n}=56)\end{array}$ & $\begin{array}{c}\text { Hypertensives } \\
(\mathrm{n}=32)\end{array}$ & \multicolumn{1}{c}{$\begin{array}{c}\text { Diabetics } \\
(\mathrm{n}=17)\end{array}$} \\
\hline Phosphatase and tensin homolog & $2.58 \pm 0.17$ & $2.78 \pm 0.29$ & $2.29 \pm 0.16$ \\
Retinol binding protein 4 & $\mathbf{2 . 3 4} \pm \mathbf{0 . 2 0}$ & $\mathbf{2 . 1 3} \pm \mathbf{0 . 1 8}$ & $\mathbf{2 . 0 8} \pm \mathbf{0 . 3 3}$ \\
Secreted frizzled-related & $3.18 \pm 0.22$ & $3.06 \pm 0.28$ & $2.91 \pm 0.33$ \\
protein 2 (sFRP2) & & & \\
Sirtuin 1 & $-2.54 \pm 0.17$ & $-2.35 \pm 0.21$ & $-3.12 \pm 0.31$ \\
Stearoyl-coenzyme A & $\mathbf{4 . 0 2} \pm \mathbf{0 . 3 5}$ & $\mathbf{3 . 2 6} \pm \mathbf{0 . 4 0}$ & $\mathbf{3 . 6 1} \pm \mathbf{0 . 5 6}$ \\
desaturase 1 & & & \\
SOCS-1 & $0.18 \pm 0.42$ & $0.37 \pm 0.47$ & $0.18 \pm 0.85$ \\
Mitochondrial superoxide & $3.48 \pm 0.27$ & $3.53 \pm 0.39$ & $3.08 \pm 0.49$ \\
dismutase-2 tv1 & & & \\
Tpl2/MAP3K8/COT-1 & $-1.48 \pm 0.24$ & $-1.46 \pm 0.26$ & $-1.74 \pm 0.30$ \\
TRB3/Tribbles 3 & $-4.33 \pm 0.19$ & $-4.65 \pm 0.22$ & $-4.52 \pm 0.27$ \\
Uncoupling protein 2 (UCP-2) & $\mathbf{1 . 3 7} \pm \mathbf{0 . 1 2}$ & $\mathbf{1 . 4 6} \pm \mathbf{0 . 1 7}$ & $\mathbf{0 . 9 5} \pm \mathbf{0 . 2 3}$ \\
Vaspin & $-1.89 \pm 0.32$ & $-1.80 \pm 0.31$ & $-2.66 \pm 0.26$ \\
VEGFA & $\mathbf{0 . 7 1} \pm \mathbf{0 . 2 2}$ & $\mathbf{0 . 5 4} \pm \mathbf{0 . 1 5}$ & $\mathbf{0 . 4 4} \pm \mathbf{0 . 2 1}$ \\
VEGFR2 & $-0.58 \pm 0.10$ & $-0.80 \pm 0.11$ & $-0.68 \pm 0.19$ \\
Zinc $\boldsymbol{\alpha}_{2}$-glycoprotein (ZAG) & $-\mathbf{0 . 5 8} \pm \mathbf{0 . 2 2}$ & $-\mathbf{0 . 6 6} \pm \mathbf{0 . 3 5}$ & $-\mathbf{0 . 9 9} \pm \mathbf{0 . 3 8}$ \\
\hline
\end{tabular}

Abbreviations: ACE, angiotensin I converting enzyme; AMPK, AMP-activated protein kinase; $B M I$, body mass index; $C D$, cluster of differentiation; CIDEA, cell death-inducing DFFA (DNA fragmentation factor-alpha)-like effector A; COT-1, cotyledon trichome 1; eNOS, endothelial nitric oxide synthase; FABP-4, fatty acid binding protein 4; Flt-1, fms-related tyrosine kinase 1; GPX3, glutathione peroxidase 3; MAP3K8, mitogen-activated protein kinase 8; MIF, macrophage migration inhibitory factor; NOX2, NADPH oxidase, PAl-1, plasminogen activator inhibitor type 1 ; PGC- $1 \alpha$, peroxisome proliferator-activated receptor gamma coactivator 1-alpha; PPAR $\gamma$, peroxisome proliferator-activated receptor-gamma; RANTES, regulated on activation, normal T cell expressed and secreted; SLAMF-1, signaling lymphocytic activation molecule family member 1 ; SOCS-1, suppresssor of cytokine signaling molecule 1; TPL-2, tumor progression locus 2; TRB-3, tribbles 3; VEGFR, vascular endothelial growth factor receptor. Hypertensives are defined as women taking drugs for treatment of hypertension and diabetics as women with fasting blood glucose values over $125 \mathrm{mg} \mathrm{dl}^{-1}$, but $71 \%$ of them were also taking anti-hypertensive drugs. The omental fat was derived from women undergoing gastric bypass surgery or lap band surgery. The values are shown as the means \pm s.e.m. of the $\Delta C p$ values from cyclophilin, the recovery standard, in omental fat with the sign reversed so that the larger the number the more mRNA. The mRNAs whose expression is at least threefold greater in fat than in non-fat/stromal cells of omental adipose tissue ${ }^{16}$ are shown in bold. Differences between controls and diabetics or hypertensives that were statistically significant with $P$-values less than 0.05 are denoted as follows: ${ }^{*} P \geqslant 0.05,{ }^{* *} P \geqslant 0.01$ and ${ }^{* * *} P \geqslant 0.001$.

The waist circumferences ranged from 69 to $180 \mathrm{~cm}$ and BMI values from 19 to $69 \mathrm{~kg} \mathrm{~m}^{-2}$ in control women undergoing gastric banding or bypass surgery or undergoing abdominoplasty a year after the initial bypass surgery. There was an excellent correlation between waist circumference and BMI (0.87 and 0.81) in control and hypertensive women, respectively (Table 1). There was also a weak but statistically significant correlation between waist circumference and circulating glucose in women taking anti-hypertensive drugs but not in control women (Table 1).

In control subjects, eight mRNAs had significant positive correlations $(P \geqslant 0.05)$ of mRNA levels with waist circumference (Table 1). I found similar positive correlations between BMI and gene expression for amyloid A, p67phox, plasminogen activator inhibitor type 1 (PAI-1) and 11ßHSD1. However, for IL-1 receptor antagonist (IL-1RA), leptin, perilipin 
Table 3 Correlation coefficients between blood glucose and mRNA expression in omental fat

\begin{tabular}{|c|c|c|c|c|c|c|}
\hline \multirow[t]{2}{*}{$\begin{array}{l}m R N A, B M I \text { or } \\
\text { waist circumference }\end{array}$} & \multicolumn{3}{|c|}{ Controls } & \multicolumn{3}{|c|}{$\begin{array}{c}\text { Subjects taking } \\
\text { anti-hypertensive agents }\end{array}$} \\
\hline & $\mathrm{n}$ & r-value & P-value & $\mathrm{n}$ & r-value & P-value \\
\hline Akt1 & 29 & -0.54 & 0.002 & 34 & -0.72 & 0.001 \\
\hline Akt2 & 44 & -0.44 & 0.003 & 41 & -0.47 & 0.002 \\
\hline CIDEA & 49 & -0.14 & 0.35 & 49 & -0.32 & 0.026 \\
\hline CD150/SLAMF1 & 41 & -0.24 & 0.14 & 43 & -0.38 & 0.013 \\
\hline Cytochrome $C$ oxidase & 35 & 0.12 & 0.47 & 39 & -0.39 & 0.013 \\
\hline GSTA4 & 29 & 0.45 & 0.014 & 30 & -0.18 & 0.34 \\
\hline $1 \alpha$ Hydroxylase & 45 & 0.37 & 0.013 & 36 & -0.16 & 0.36 \\
\hline PGC- $1 \alpha$ & 28 & -0.38 & 0.045 & 37 & 0.07 & 0.68 \\
\hline SIRT1 & 36 & -0.58 & 0.001 & 28 & -0.31 & 0.11 \\
\hline TLR4 & 42 & -0.15 & 0.34 & 42 & -0.32 & 0.040 \\
\hline VEGFR1 & 44 & -0.58 & 0.001 & 45 & -0.06 & 0.67 \\
\hline Waist circumference & 54 & 0.20 & 0.15 & 54 & 0.27 & 0.04 \\
\hline BMI & 56 & 0.22 & 0.11 & 54 & 0.38 & 0.004 \\
\hline
\end{tabular}

Abbreviations: BMI, body mass index; $C D$, cluster of differentiation; CIDEA, cell death-inducing DFFA (DNA fragmentation factor-alpha)-like effector $A$; GSTA4, glutathione S-transferase 4; PGC- $1 \alpha$, peroxisome proliferator-activated receptor gamma coactivator 1-alpha; SIRT-1, sirtuin 1; SLAMF-1, signaling lymphocytic activation molecule family member 1 ; TLR-4, Toll-like receptor 4; VEGFR, vascular endothelial growth factor receptor; $1 \alpha$ hydroxylase; $1 \alpha, 25$-dihydroxyvitamin $\mathrm{D}$ hydroxylase. The correlation between fasting blood glucose levels and the expression of all the mRNAs shown in Table 2 was examined, but only those with statistically significant correlations are shown $(P \geqslant 0.05)$ between blood glucose and the mRNAs in Table 2 in either control subjects or subjects taking anti-hypertensive drugs. Omental fat was derived from women undergoing gastric bypass surgery, lap band surgery or abdominoplasy. The controls included those diabetics not taking hypertensive drugs. The subjects on anti-hypertensive drugs included diabetics. The mRNAs whose expression is at least threefold greater in fat than in non-fat/stromal cells of omental adipose tissue ${ }^{16}$ are shown in bold.

and PI-3 kinase, no statistically significant correlation was seen between their gene expression and BMI. Interestingly in the subjects taking anti-hypertensive drugs there was no statistically significant correlation between waist circumference or BMI and mRNA levels of any of these six except for IL-1 Ra.

Of the mRNAs with significant correlations between waist circumference and mRNA expression in omental fat of control subjects, five of these (leptin, 11ßHSD1, amyloid A, perilipin, and TLR4 are preferentially enriched in adipocytes/fat cells by 12-fold or more as compared with non-fat/ stromal cells of human adipose tissue. ${ }^{16}$ Significant correlations were also seen for four proteins whose mRNAs are expressed equally in all cells (IL-1RA and PI-3 kinase catalytic subunit) or primarily in the non-fat/stromal cells (p67phox, PAI-1) of human omental adipose tissue. ${ }^{16}$

The mRNA expression of TLR4 negatively correlated with both waist circumference and BMI, while CIDEA negatively correlated with BMI (Table 1). In contrast to what was seen with regard to p67phox, PAI-1, 11ßHSD1, IL-1RA, leptin and perilipin where the correlation coefficients were higher based on waist circumference rather than BMI, the opposite was seen for CIDEA (Table 1). The correlation coefficient for
Table 4 Pearson correlations between waist circumference and levels of 18 serum proteins

\begin{tabular}{|c|c|c|c|c|c|c|}
\hline \multirow[t]{2}{*}{ Adipokine } & \multicolumn{3}{|c|}{ Controls } & \multicolumn{3}{|c|}{$\begin{array}{c}\text { Women taking } \\
\text { anti-hypertensive drugs }\end{array}$} \\
\hline & $n$ & r-value & P-value & $\mathrm{n}$ & r-value & P-value \\
\hline FABP4 & 13 & 0.88 & 0.0001 & 10 & 0.46 & 0.18 \\
\hline Adipsin & 12 & 0.71 & 0.009 & 10 & 0.22 & 0.53 \\
\hline$s P L A_{2}$ & 13 & 0.52 & 0.06 & 10 & 0.58 & 0.07 \\
\hline IL-1 RA & 23 & 0.51 & 0.01 & 10 & -0.14 & 0.71 \\
\hline IL-8 & 13 & 0.41 & 0.16 & 10 & -0.24 & 0.50 \\
\hline GPX-3 & 23 & 0.40 & 0.06 & 10 & 0.14 & 0.70 \\
\hline Lipocalin-2 & 13 & 0.40 & 0.17 & 10 & -0.01 & 0.97 \\
\hline PAI-1 & 13 & 0.40 & 0.11 & 10 & -0.02 & 0.94 \\
\hline sFlt1 & 13 & 0.39 & 0.19 & 10 & -0.15 & 0.67 \\
\hline RANTES & 13 & 0.37 & 0.21 & 10 & 0.27 & 0.46 \\
\hline Leptin & 19 & 0.36 & 0.13 & 11 & 0.77 & 0.005 \\
\hline NGF & 16 & 0.20 & 0.45 & 10 & -0.44 & 0.21 \\
\hline MCP-1 & 23 & 0.19 & 0.39 & 10 & 0.01 & 0.97 \\
\hline IL-10 & 23 & 0.07 & 0.73 & 10 & -0.24 & 0.51 \\
\hline OPG & 12 & 0.06 & 0.85 & 10 & 0.42 & 0.43 \\
\hline CD14 & 23 & -0.03 & 0.89 & 10 & -0.12 & 0.73 \\
\hline CD163 & 23 & -0.19 & 0.38 & 10 & 0.03 & 0.92 \\
\hline ZAG & 13 & -0.26 & 0.38 & 10 & 0.44 & 0.20 \\
\hline
\end{tabular}

Abbreviations: $C D$, cluster of differentiation; FABP-4, fatty acid binding protein 4; GPX3, glutathione peroxidase 3; IL-1RA, interleukin 1 receptor antagonist; IL-1RA, interleukin (IL)-1 receptor antagonist; MCP-1, monocyte chemoattractant protein 1; NGF, nerve growth factor; OPG, osteoprotegerin; PAI-1, plasminogen activator inhibitor type 1 ; RANTES, regulated on activation, normal T cell expressed and secreted; sFlt-1, soluble fms-like tyrosine kinase-1; sPLA 2 , soluble phospholipase $A_{2} ; Z A G$, zinc $\alpha 2$-glycoprotein. Proteins were analyzed in serum from the same 23 subjects, except for leptin, GPX3, IL-10, MCP-1, NGF, CD14, CD163, IL-1Ra and MCP-1, where serums from 10 additional subjects were examined. The Pearson correlation coefficient $(r)$ is given along with the $P$-value for the correlation between circulating levels of the proteins and waist circumference. All subjects were undergoing gastric bypass or lap band surgery with a mean waist circumference of $131 \mathrm{~cm}$ (range from 107 to $168 \mathrm{~cm}$ ). The mRNAs whose expression is at least threefold greater in fat than in non-fat/stromal cells of omental adipose tissue ${ }^{16}$ are shown in bold.

CIDEA against waist circumference was only -0.27 and marginally significant while it was -0.37 against BMI and was highly significant. Among the 96 mRNAs with no significant correlation in mRNA expression to either waist circumference or BMI are the 56 mRNAs shown in Table 2.

Effect of diabetes and the administration of anti-hypertensive drugs on gene expression

Table 2 shows the results using omental adipose tissue from control women, women taking anti-hypertensive agents who were not diabetic and diabetics either taking insulin or oral agents with fasting blood glucose values above $125 \mathrm{mg} \mathrm{dl}^{-1}$. The average BMI of the control women was $49.1 \mathrm{~kg} \mathrm{~m}^{-2}$, that of the patients taking anti-hypertensive drugs was $48.5 \mathrm{~kg} \mathrm{~m}^{-2}$ and for the diabetics was $54.2 \mathrm{~kg} \mathrm{~m}^{-2}$, and all fat samples were from extremely obese women undergoing gastric bypass or lap band surgery. The women taking only anti-hypertensive drugs did not differ from the control women except for significantly lower fasting blood glucose values (Table 2). Although, the diabetic women were 
significantly older than the control women (Table 2), there was no correlation between age and the expression of any of the mRNAs shown in Table 2 (data not shown). The waist circumferences of the diabetic women was 3.9\% greater than those of the controls but not statistically significant (Table 2).

The control women who were taking only anti-hypertensive medications had 100\% higher levels of CD150/signaling lymphocytic activation molecule family member 1 , a $52 \%$ higher expression of renin receptor mRNA but a 32\% lower expression of apelin and a 28\% lower expression of VEGFR1 (Table 2). In the women with diabetes, there was a $42 \%$ decrease in Akt1, a 53\% decrease in Akt2, a $67 \%$ decrease in TLR4, a 50\% decrease in VEGFR1, a $29 \%$ decrease in p67 phox mRNA expression and a $26 \%$ lower expression of VEGFR1 plus fms-related tyrosine kinase 1 in omental fat (Table 2). There were only four diabetic women not taking anti-hypertensives. Only in the case of VEGFR1 were statistically significant effects seen in both diabetic and hypertensive control patients. The decrease in VEGFR1 was $40 \%$ greater in the four diabetic women not taking antihypertensive drugs.

The relationship in obese women undergoing gastric bypass or lap band surgery between their fasting blood glucose values and gene expression in omental fat was examined in the studies shown in Table 3. In these studies, as shown in Table 1, the diabetic women were divided between the control and hypertension groups. The diabetic women not taking anti-hypertensive drugs were combined with the controls. The Pearson correlation coefficients were determined for all the mRNAs shown in Table 2, but only those with statistically significant correlations are shown in Table 3. In the women not taking anti-hypertensives there was a significant positive correlation between circulating glucose and mRNA expression in omental fat for glutathione $S$-transferase A 4 and $1 \alpha$-hydroxylase. There were significant negative correlations between blood glucose and gene expression for Akt1, Akt2, VEGFR1, sirtuin-1, PI-3 kinase and peroxisome proliferator activator receptor- $\gamma$ coactivator $1 \alpha$ in control subjects (Table 3 ). In subjects taking antihypertensive drugs, the expression of none of these mRNAs was significantly affected by the blood glucose concentration except for Akt1 and Akt2 where the correlation coefficients were even greater. However, the mRNA expression of TLR4, CD150/SLAMF1, cytochrome $C$ oxidase and CIDEA had significant negative correlations, with circulating blood glucose in women taking anti-hypertensive drugs (Table 3).

Effect of waist circumference and BMI on circulating levels of 18 putative adipokines

Three of the mRNAs whose expression in omental fat positively correlated with waist circumference in control subjects also circulate as adipokines (leptin, IL-1RA and plasminogen activator inhibitor 1 ). The circulating levels of these adipokines along with those of 15 other serum proteins whose circulating levels have been reported to be affected by obesity were correlated with waist circumference of both control women and those taking anti-hypertensive drugs (Table 4). The correlation between waist circumference and mRNA expression in omental fat was also examined for the 15 proteins shown in Table 1 but no statistically significant correlations were seen (data not shown). The circulating levels of FABP4, IL-1RA and adipsin significantly correlated with waist circumference with Pearson correlation coefficients of 0.51 or greater in control subjects (Table 4 ). There were correlation coefficients of 0.40 or greater for PAI-1, lipocalin-2, glutathione peroxidase 3 and IL-1RA but they were not statistically significant because of the low number of control subjects. In the control women, there were similar positive correlations between waist circumference and mRNA expression in omental fat and the circulating levels, only for IL-1RA (Tables 1 and 4).

There were no significant correlations between waist circumference and the circulating levels of adipokines in the women taking anti-hypertensive drugs except with regard to leptin where the correlation coefficient was actually higher and statistically significant as compared with circulating levels in the control subjects (Table 4). Soluble $\mathrm{PLA}_{2}$ was the only circulating adipokine whose correlation coefficients were similar in both the control and hypertensive women. There was no statistically significant correlation between age or blood glucose and circulating levels of PLA, FABP4, adipsin or IL-1RA (data not shown).

\section{Discussion}

Waist circumference is arguably the best inexpensive marker for visceral obesity. ${ }^{6}$ It appears to be better marker than BMI in the sense that more of the mRNAs examined in this observational report using human omental fat showed greater positive correlations between their gene expression and waist circumference as compared with BMI. The reasons for these differences are unclear and just the opposite was seen with regard to CIDEA whose gene expression better correlated with BMI than with waist circumference. What is interesting about the current findings is that there are mRNAs affected by hypertension as well as by diabetes and all are different from those affected by obesity. It should be noted that the definition of diabetes is fasting blood glucose values over $125 \mathrm{mg} \mathrm{dl}^{-1}$ and these values presumably reflect the insulin resistance that cannot be adequately compensated for by enhanced release of insulin.

The present observational studies are cross-sectional in nature and can demonstrate correlations but not causation. A further complication is that in patients taking antihypertensive drugs, it is possible that the differences are due to the drugs or unknown confounding variables. Prospective studies will be needed to determine whether similar changes are seen in extremely obese patients with 
hypertension both, before and after treatment with antihypertensive agents and to correlate changes in blood pressure with gene expression in omental fat which was not performed in the present studies. The present results suggest that hypertension profoundly affects omental fat gene expression, but this conclusion is based on observational studies. The possibility must be considered that the effects may be the result of some uncontrolled confounding variable.

Another problem is that many of the mRNAs whose expression was altered in omental fat by hypertension or diabetes are found in all cells or preferentially enriched in the non-fat/stromal cells of omental adipose tissue from very obese women. ${ }^{16}$ Of the mRNAs whose expression correlated with circulating blood glucose in controls, only Akt2 is preferentially found in fat cells (fourfold higher levels in fat over non-fat cells). ${ }^{16}$ None of the mRNAs whose expression in omental fat was altered in patients taking only antihypertensive agents are enriched in fat cells except for CIDEA. It would be expected that obesity would preferentially affect gene expression in fat cells as they are markedly larger. However, the expression of p67phox, PI-3 kinase, PAI1 and IL-1RA in omental fat positively correlated with waist circumference but these mRNAs are not enriched in human omental adipocytes/fat cells. This probably reflects enhanced accumulation of macrophages and other inflammatory cells in omental adipose tissue, as their accumulation is positively linked to fat mass in humans. ${ }^{17,18}$ However, why positive correlations between waist circumference and the accumulation in omental adipose tissue of two of these inflammatory markers (p67phox subunit of NADPH oxidase and PAI-1) were abolished in patients taking anti-hypertensive drugs is unclear. The positive correlation between waist circumference and IL-1RA gene expression in omental fat was the same in women taking anti-hypertensive drugs, suggesting that IL-1RA is an obesity marker unaffected by hypertension.

The circulating level of leptin is upregulated in obesity ${ }^{19}$ as is that of adipsin. ${ }^{20}$ Similarly $\mathrm{Xu}$ et al ${ }^{21}$ reported a positive correlation between BMI and circulating FABP4, and Weyer et al. $^{22}$ reported a positive correlation between BMI and circulating $\mathrm{SPLA}_{2}$. A significant correlation was seen between waist circumference, both for circulating levels of IL-1RA and its gene expression in omental fat but not for FABP4, PLA2 or adipsin in control subjects. These data indicate that gene expression in omental fat correlates with circulating levels for IL-1RA but not for all adipokines. However, it should be recognized that the levels of protein expression in the omental fat were not measured and mRNA levels may not necessarily translate into altered levels of protein. Furthermore, the circulating levels of adipokines are probably regulated to a greater extent by subcutaneous than omental fat because of the greater mass of subcutaneous fat.

The present finding confirms the previously reported positive correlation coefficient between waist circumference or BMI and gene expression of $11 \beta \mathrm{HSD} 1 .^{23-25}$ Leptin, amyloid A, perilipin and $11 \beta \mathrm{HSD} 1$ are all preferentially expressed in the fat rather than the non-fat/stromal cells of adipose tissue ${ }^{16}$ and whether their increased expression in obesity reflects anything more than an expansion of fat cells is unclear. Veilleux et al. ${ }^{26}$ have reported that human omental adipocyte size correlated with $11 \beta \mathrm{HSD} 1$ activity. This protein has been postulated to be an important regulator at the interface of obesity and inflammation, as it catalyzes the formation of cortisol from cortisone. ${ }^{27}$ The effects of glucocorticoids are complex, as they both suppress the initiation of inflammation and can cause symptoms identical to those seen insulin resistance and hypertension. ${ }^{27}$ It is an attractive hypothesis that obesity promotes local glucocorticoid formation in adipose tissue and that this is linked to insulin resistance.

IL-1RA, $1 \alpha$-hydroxylase, p67phox, macrophage migration inhibitory factor, PR domain-containing 16, endothelin-1, PAI-1, IL-1RA and VEGFR/fms-related tyrosine kinase 1 are either expressed to the same extent in all cells or preferentially expressed in the non-fat/stromal cells of human omental fat. ${ }^{16}$ They are probably made and released primarily by macrophages, endothelial and other non-fat cells present in omental fat. The elevated expression of IL-1RA, p67phox and PAI-1 in omental fat of obese individuals supports the hypothesis that obesity is associated with an inflammatory response, as some of these factors are known inflammatory mediators. We conclude that the p67phox subunit of NADPH oxidase, PAI-1 and IL-1RA are more important in the inflammatory response of visceral adipose tissue seen in extreme obesity than adipokines such as IL-8, IL-6, tumor necrosis factor- $\alpha$, visfatin, osteopontin, IL-1 $\beta$, RANTES, vaspin, apelin and lipocalin-2 whose expression was not altered in extremely obese women. The p67phox protein is required for activation of the superoxide-producing NADPH oxidase in phagocytes, ${ }^{28}$ and thus has a key role in the generation of reactive-oxygen species that are a hallmark of inflammation. The circulating levels of IL-1RA ${ }^{29}$ as well as its gene expression and protein content of subcutaneous fat are elevated in obese humans. ${ }^{30,31}$ Interestingly, IL-1RA was the only mRNA in omental fat whose expression correlated with both waist circumference and circulating glucose in women taking hypertensive drugs. It is generally accepted that IL-1RA is anti-inflammatory adipokine released at much higher levels by adipose tissue than the more familiar pro-inflammatory adipokines. ${ }^{29-31}$ The stimulus for its release is unclear but could involve leptin or inflammatory adipokines. ${ }^{29-31}$ The finding that PAI-1 expression in omental fat positively correlated with obesity confirms the report of Alessi et al. ${ }^{32}$ who measured the protein content of both human omental and subcutaneous fat. PAI-1 is a member of the serpin family that inhibits the fibrinolytic system and has been postulated to have a role in the development of inflammation that is seen in obesity. ${ }^{33}$

There is evidence that the renin-angiotensin system is involved in the development of hypertension. ${ }^{34-36}$ Furthermore, the renin-angiotensin system is present in fat 
cells, $^{35}$ and I examined the five main proteins of this system in omental fat: angiotensin II receptor-2, angiotensin II receptor-1, the renin receptor, angiotensingen and angiotensin I converting enzyme. In omental fat, only angiotensinogen mRNA is preferentially found in fat cells. ${ }^{16}$ The only component of renin-angiotensin system that was statistically enhanced in omental fat of patients taking antihypertensive drugs was the renin receptor and it showed a $52 \%$ increase in its gene expression. It has been shown that the renin receptor is preferentially localized in the non-fat cells of human fat, specifically in vascular structures. ${ }^{37}$ The significance of its increased expression in patients taking anti-hypertensive drugs is unclear. It could be a response to these drugs. The only other mRNA whose expression was enhanced in omental fat of hypertensives was CD150/ SLAMF1, which was enhanced by $100 \%$. CD150/SLAMF1 is a signaling lymphocytic activation molecule that is a cellsurface receptor on hematopoietic cells such as T or B cells, ${ }^{38}$ but the link between its known effects and hypertension remains to be established.

The $32 \%$ reduction in apelin gene expression in omental fat of patients taking anti-hypertensive drugs is in agreement with findings that hypertension reduces the circulating levels of apelin in humans. ${ }^{39,40}$ Furthermore, systemic administration of apelin inhibits the antidiuretic effects of arginine vasopressin, decreases blood pressure and improves cardiac contractility. ${ }^{41}$ The present studies in massively obese women suggest that future investigations should consider the roles of apelin, renin receptor, Akt2, TLR4, cytochrome $C$ oxidase, retinol binding protein 4, CIDEA, IL-1RA and CD150/SLAMF1 in the blood vessels, macrophages and/or other non-fat cells of human visceral omental adipose tissue with regard to hypertension.

The studies suggest that the effects of diabetes on omental fat are secondary to the insulin resistance as reflected by elevations in the circulating blood glucose. This conclusion is based on the high degree of inverse correlation between blood glucose values and the expression of mRNAs such as Akt1, Akt2, sirtuin-1, PI-3 kinase, peroxisome proliferator activator receptor- $\gamma$ coactivator $1 \alpha$ and VEGFR1 in omental fat. It is also unclear why only in control women, significant positive correlation was seen between blood glucose and glutathione $S$-transferase A4 as well as $1 \alpha$-hydroxylase gene expression, but none of these mRNAs were affected by obesity in human omental fat. Glutathione $S$-transferase is involved in lipid aldehyde detoxification and an increase in its activity should reduce the harmful effects of reactive oxygen species formation seen in insulin resistance. ${ }^{42}$ Although the $1 \alpha$-hydroxylase enzyme has been found in adipose tissue, its role in the formation of vitamin D3 in humans is unclear. High vitamin D intake has been associated with insulin resistance. ${ }^{43}$

It is possible that elevations in blood glucose and changes in omental fat gene expression are both secondary to insulin resistance. The reduction in gene expression of Akt1 and Akt2 as circulating glucose increases would agree with a reduction in insulin action. What is interesting is the hypothesis that elevated levels of free fatty acids, purported to be elevated in obesity, could through activation of the TLR4 receptor inhibit insulin action. ${ }^{44}$ Thus, a reduction in TLR4 message might be part of a feedback loop to restore insulin sensitivity, while the reduction of Akt1 might be a direct effect of an enhanced inflammatory response at high glucose levels.

The protein kinases, Akt1 and Akt2 are key factors in the insulin stimulation of glucose metabolism and inhibition of both blocks insulin action in 3T3-L1 adipocytes. ${ }^{45}$ Which one is more important is unclear, as fatty acid infusion into rats selectively impaired Akt1, but not Akt2 activation by insulin in rat muscles. ${ }^{46}$ In contrast, in skeletal muscles from obese humans, the activation by insulin of Akt1 was unimpaired, whereas that of Akt 2 was inhibited as compared with the increases seen in muscle from lean humans. ${ }^{47}$ The present results suggest that both kinases are involved, with Akt2 being found primarily in fat cells whereas Akt1 is found in all cells.

The role of VEGFR1 in insulin action is unknown. Some effects of VEGF may be mediated through the VEGFR1 receptor but the primary role of VEGFR1 is currently thought to be as the precursor of a decoy receptor for VEGF formed by removing the transmembrane spanning unit, resulting in release of a protein called soluble fms-like tryrosine kinase that binds to and inactivates VEGF. ${ }^{48}$

In conclusion, obesity in women, as measured by waist circumference, correlated with increased expression of inflammatory adipokines such as p67phox, PAI-1 and IL-1RA in omental fat. Downregulation of CIDEA was seen in obesity, of apelin and VEGFR1 in women taking antihypertensive drugs and of Akt1, Akt2, TLR4 and VEGFR1 in diabetic women. In contrast, the mRNA expression of CD150/SLAMF1 and the renin receptor was enhanced in omental fat from women taking anti-hypertensive drugs. These results indicate that in omental fat, the responses seen in hypertensive and diabetic patients are different from those seen with obesity. Future work should determine whether these changes in mRNA expression are reflected in protein expression and the role of Akt1/2 and TLR 4 in the development of diabetes in obese women.

\section{Conflict of interest}

The author declares no conflict of interest.

\section{Acknowledgements}

This study was funded in part by the Van Vleet Chair of Excellence, University of Tennessee and Zen-Bio Inc. I am indebted to Dr Atul Madan and Dr David Tichansky of the Department of Surgery, for provision of the adipose tissue, and to Mrs Paramjeet Cheema for her technical assistance. 


\section{References}

1 Pories WJ. Bariatric surgery: risks and rewards. J Clin Endocrinol Metab 2008; 93: 589-596.

2 Bult MJ, van Dalen T, Muller AF. Surgical treatment of obesity. Eur J Endocrinol 2008; 158: 135-145.

3 Livingston EH, Ko CY. Effect of diabetes and hypertension on obesity-related mortality. Surgery 2005; 137: 16-25.

4 Karelis AD, Faraj M, Bastard JP, St-Pierre DH, Brochu M, Prud'homme $\mathrm{D}$ et al. The metabolically healthy but obese individual presents a favorable inflammation profile. J Clin Endocrinol Metab 2005; 90: 4145-4150.

5 Despres JP, Lemieux I. Abdominal obesity and metabolic syndrome. Nature 2006; 444: 881-887.

6 Scherzer R, Shen W, Bacchetti P, Kotler D, Lewis CE, Shlipak MG et al. Simple anthropometric measures correlate with metabolic risk indicators as strongly as magnetic resonance imagingmeasured adipose tissue depots in both HIV-infected and control subjects. Am J Clin Nutr 2008; 87: 1809-1817.

7 Shen W, Punyanitya M, Chen J, Gallagher D, Albu J, Pi-Sunyer X et al. Waist circumference correlates with metabolic syndrome indicators better than percentage fat. Obesity 2006; 14: 727-736.

8 Canoy D, Boekholdt SM, Wareham N, Luben R, Welch A, Bingham $\mathrm{S}$ et al. Body fat distribution and risk of coronary heart disease in men and women in the European prospective investigation into cancer and nutrition Norfolk cohort: a population-based prospective study. Circulation 2007; 116: 2933-2943.

9 Despres JP, Lemieux I, Bergeron J, Pibarot P, Mathieu P, Larose E et al. Abdominal obesity and the metabolic syndrome: contribution to global cardiometabolic risk. Arterioscler Thromb Vasc Biol 2008; 28: 1039-1049.

10 Pischon T, Boeing H, Hoffmann K, Bergmann M, Schulze MB, Overvad $\mathrm{K}$ et al. General and abdominal adiposity and the risk of death in Europe. N Engl J Med 2008; 359: 2105-2120.

11 Madan AK, Tichansky DS, Coday M, Fain JN. Comparison of IL-8, IL-6 and $\mathrm{PGE}_{2}$ formation by visceral (omental) adipose tissue of obese Caucasian as compared to African-American women. Obes Surg 2006; 16: 1342-1350.

12 Platell C, Cooper D, Papadimitriou JM, Hall JC. The omentum. World J Gastroenterol 2000; 6: 169-176.

13 Thorne A, Lonnqvist F, Apelman J, Hellers G, Arner P. A pilot study of long-term effects of a novel obesity treatment: omentectomy in connection with adjustable gastric banding. Intl J Obes 2002; 26: 193-199.

14 Fain JN, Buehrer B, Bahouth SW, Tichansky DS, Madan AK. Comparison of messenger RNA distribution for 60 proteins in fat cells vs the nonfat cells of human omental adipose tissue. Metabolism 2008; 57: 1005-1015.

15 Bustin SA. Absolute quantification of mRNA using real-time reverse transcription polymerase chain reaction assays. $J \mathrm{Mol}$ Endocrinol 2000; 25: 169-193.

16 Fain JN. Release of inflammatory mediators by human adipose tissue is enhanced in obesity and primarily by the nonfat cells: a review. Mediators Inflamm 2010; 2010: 513948.

$17 \mathrm{Xu} \mathrm{H}$, Barnes GT, Yang Q, Tan G, Yang D, Chou CJ et al. Chronic inflammation in fat plays a crucial role in the development of obesity-related insulin resistance. J Clin Invest 2003; 112: 1821-1830.

18 Weisberg SP, McCann D, Desai M, Rosenbaum M, Leibel RL, Ferrante Jr AW. Obesity is associated with macrophage accumulation in adipose tissue. J Clin Invest 2003; 112: 1796-1808.

19 van Dielen FM, van't Veer C, Schols AM, Soeters PB, Buurman WA, Greve JW. Increased leptin concentrations correlate with increased concentrations of inflammatory markers in morbidly obese individuals. Int J Obes Relat Metab Disord 2001; 25: 1759-1766.

20 Napolitano A, Lowell BB, Damm D, Leibel RL, Ravussin E, Jimerson DC et al. Concentrations of adipsin in blood and rates of adipsin secretion by adipose tissue in humans with normal, elevated and diminished adipose tissue mass. Int $J$ Obes Relat Metab Disord 1994; 18: 213-218.

21 Xu A, Wang Y, Xu JY, Stejskal D, Tam S, Zhang J et al. Adipocyte fatty acid-binding protein is a plasma biomarker closely associated with obesity and metabolic syndrome. Clin Chem 2006; 52: 405-413.

22 Weyer C, Yudkin JS, Stehouwer CD, Schalkwijk CG, Pratley RE, Tataranni PA. Humoral markers of inflammation and endothelial dysfunction in relation to adiposity and in vivo insulin action in Pima Indians. Atherosclerosis 2002; 161: 233-242.

23 Rask E, Walker BR, Soderberg S, Livingstone DE, Eliasson M, Johnson $\mathrm{O}$ et al. Tissue-specific changes in peripheral cortisol metabolism in obese women: increased adipose $11 \beta$-hydroxysteroid dehydrogenase type 1 activity. J Clin Endocrinol Metab 2002; 87: 3330-3336.

24 Michailidou Z, Jensen MD, Dumesic DA, Chapman KE, Seckl JR, Walker BR et al. Omental 11ß-hydroxysteroid dehydrogenase 1 correlates with fat cell size independently of obesity. Obesity 2007; 15: 1155-1163.

25 Desbriere R, Vuaroqueaux V, Achard V, Boullu-Ciocca S, Labuhn M, Dutour A et al. 11ß-hydroxysteroid dehydrogenase type 1 mRNA is increased in both visceral and subcutaneous adipose tissue of obese patients. Obesity 2006; 14: 794-798.

26 Veilleux A, Rheaume C, Daris M, Luu-The V, Tchernof A. Omental adipose tissue type $111 \beta$-hydroxysteroid dehydrogenase oxoreductase activity, body fat distribution, and metabolic alterations in women. J Clin Endocrinol Metab 2009; 94: 3550-3557.

27 Staab CA, Maser E. 11ß-hydroxysteroid dehydrogenase type 1 is an important regulator at the interface of obesity and inflammation. J Steroid Biochem Mol Biol 2010; 119: 56-72.

28 Yuzawa S, Miyano K, Honbou K, Inagaki F, Sumimoto H. The domain organization of $\mathrm{p} 67$ phox, a protein required for activation of the superoxide-producing NADPH oxidase in phagocytes. J Innate Immun 2009; 1: 543-555.

29 Meier CA, Bobbioni E, Gabay C, Assimacopoulos-Jeannet F, Golay A, Dayer JM. IL-1 receptor agonist serum levels are increased in human obesity: a possible link to the resistance to leptin? J Clin Endocrinol Metab 2002; 87: 1184-1188.

30 Juge-Aubry CE, Somm E, Giusti V, Pernin A, Chicheportiche R, Verdumo C et al. Adipose tissue is a major source of interleukin-1 receptor antagonist: upregulation in obesity and inflammation. Diabetes 2003; 52: 1104-1110.

31 Dayer JM, Chicheportiche R, Juge-Aubry C, Meier C. Adipose tissue has anti-inflammatory properties: focus on IL-1 receptor antagonist (IL-1Ra). Ann NY Acad Sci 2006; 1069: 444-453.

32 Alessi MC, Bastelica D, Morange P, Berthet B, Leduc I, Verdier M et al. Plasminogen activator inhibitor 1 , transforming grown factor- $\beta_{1}$, and BMI are closely associated in human adipose tissue during morbid obesity. Diabetes 2000; 49: 1374-1380.

33 Alessi MC, Poggi M, Juhan-Vague I. Plasminogen activator inhibitor-1, adipose tissue and insulin resistance. Curr Opin Lipidol 2007; 18: 240-245.

34 Leiter LA, Lewanczuk RZ. Of the renin-angiotensin system and reactive oxygen species: type 2 diabetes and angiotensin II inhibition. Am J Hypertens 2005; 18: 121-128.

35 Cassis LA, Police SB, Yiannikouris F, Thatcher SE. Local adipose tissue renin-angiotensin system. Curr Hypertens Rep 2008; 10: 93-98.

36 Yvan-Charvet L, Massiera F, Lamande N, Ailhaud G, Teboul M, Moustaid-Moussa $\mathrm{N}$ et al. Deficiency of angiotensin type 2 receptor rescues obesity but not hypertension induced by overexpression of angiotensin in adipose tissue. Endocrinology 2009; 150: $1421-1428$.

37 Achard V, Boullu-Ciocca S, Desbriere R, Nguyen G, Grino M. Renin receptor expression in human adipose tissue. Am J Physiol Regul Integr Comp Physiol 2007; 292: R274-R282.

38 Detre C, Keszei M, Romero X, Tsokos GC, Terhorst C. SLAM family receptors and the SLAM-associated protein (SAP) 
modulate $\mathrm{T}$ cell functions. Semin Immunopathol 2010; 32: $157-171$

39 Przewlocka-Kosmala M, Kotwica T, Mysiak A, Kosmala W. Reduced circulating apelin in essential hypertension and its association with cardiac dysfunction. J Hypertens 2011; 29: 971-979.

40 Sonmez A, Celebi G, Erdem G, Tapan S, Genc H, Tasci I et al. Plasma apelin and ADMA levels in patients with essential hypertension. Clin Exp Hypertens 2010; 32: 179-183.

41 Llorens-Cortes C, Kordon C. Jacques Benoit lecture: the neuroendocrine view of the angiotensin and apelin systems. J Neuroendocrinol 2008; 20: 279-289.

42 Curtis JM, Grimsrud PA, Wright WS, Xu X, Foncea RE, Graham DW et al. Downregulation of adipose glutathione $S$-transferase A4 leads to increased protein carbonylation, oxidative stress, and mitochondrial dysfunction. Diabetes 2010; 59: $1132-1142$.

$43 \mathrm{Li} \mathrm{J}$, Byrne ME, Chang E, Jiang Y, Donkin SS, Buhman KK et al. $1 \alpha, 25$-Dihydroxyvitamin D hydroxylase in adipocytes. I Steroid Biochem Mol Biol 2008; 112: 122-126.

44 Kim JJ, Sears DD. TLR4 and insulin resistance. Gastroenterol Res Pract 2010; 2010: pii: 212563.
45 Jiang ZY, Zhou QL, Coleman KA, Chouinard M, Boese Q Czech MP. Insulin signaling through Akt/protein kinase B analyzed by small interfering RNA-mediated gene silencing. Proc Natl Acad Sci USA 2003; 100: 7569-7574.

46 Kim YB, Shulman GI, Kahn BB. Fatty acid infusion selectively impairs insulin action on Akt1 and protein kinase C lambda/zeta but not on glycogen synthase kinase 3. J Biol Chem 2002; 277: 32915-32922.

47 Brozinick Jr JT, Roberts BR, Dohm GL. Defective signaling through Akt- 2 and -3 but not Akt-1 in insulin-resistant human skeletal muscle: potential role in insulin resistance. Diabetes 2003; 52: 935-941.

48 Tam J, Duda DG, Perentes JY, Quadri RS, Fukumura D, Jain RK. Blockade of VEGFR2 and not VEGFR1 can limit diet-induced fat tissue expansion: role of local versus bone marrow-derived endothelial cells. PLoS One 2009; 4: e4974.

This work is licensed under the Creative Commons Attribution-NonCommercial-No Derivative Works 3.0 Unported License. To view a copy of this license, visit http://creativecommons.org/ licenses/by-nc-nd/3.0/ 